\title{
ARTICLE OPEN \\ Transcriptional responses of Candida glabrata biofilm cells to fluconazole are modulated by the carbon source
}

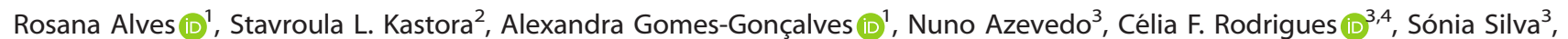 \\ Liesbeth Demuyser, ${ }^{5,6}$, Patrick Van Dijck $\mathbb{1}^{5,6}$, Margarida Casal ${ }^{1}$, Alistair J. P. Brown ${ }^{2,7}$, Mariana Henriques ${ }^{3}$ and Sandra Paiva (1) ${ }^{1 *}$
}

Candida glabrata is an important human fungal pathogen known to trigger serious infections in immune-compromised individuals. Its ability to form biofilms, which exhibit high tolerance to antifungal treatments, has been considered as an important virulence factor. However, the mechanisms involving antifungal resistance in biofilms and the impact of host niche environments on these processes are still poorly defined. In this study, we performed a whole-transcriptome analysis of $C$. glabrata biofilm cells exposed to different environmental conditions and constraints in order to identify the molecular pathways involved in fluconazole resistance and understand how acidic $\mathrm{pH}$ niches, associated with the presence of acetic acid, are able to modulate these responses. We show that fluconazole treatment induces gene expression reprogramming in a carbon source and pH-dependent manner. This is particularly relevant for a set of genes involved in DNA replication, ergosterol, and ubiquinone biosynthesis. We also provide additional evidence that the loss of mitochondrial function is associated with fluconazole resistance, independently of the growth condition. Lastly, we propose that C. glabrata Mge1, a cochaperone involved in iron metabolism and protein import into the mitochondria, is a key regulator of fluconazole susceptibility during carbon and $\mathrm{pH}$ adaptation by reducing the metabolic flux towards toxic sterol formation. These new findings suggest that different host microenvironments influence directly the physiology of C. glabrata, with implications on how this pathogen responds to antifungal treatment. Our analyses identify several pathways that can be targeted and will potentially prove to be useful for developing new antifungals to treat biofilm-based infections.

npj Biofilms and Microbiomes (2020)6:4; https://doi.org/10.1038/s41522-020-0114-5

\section{INTRODUCTION}

Candida spp. are important fungal pathogens known to trigger serious infections in immune-compromised individuals, affecting billions of people every year. ${ }^{1}$ While C. albicans has been predominantly identified as the most common cause of candidiasis, infections caused by non-albicans Candida strains such as C. glabrata have been increasing worldwide. ${ }^{2,3}$ This trend has coincided with the prophylactic use of antifungals, which has resulted in increased drug resistance. ${ }^{4}$ Moreover, C. glabrata has the ability to adhere and to form biofilms on both biotic and abiotic surfaces, such as host tissues and implanted medical devices. ${ }^{5-8}$ This ability makes these infections a clinical challenge, as the cells in biofilms are also intrinsically resistant to conventional antifungal treatments. ${ }^{9}$

In order to survive and successfully proliferate in the different host niches, C. glabrata must rapidly adapt to a diverse range of environmental stresses, such as temperature, $\mathrm{pH}$ fluctuations, and nutrient availability. Some of these niches are complex, dynamic and frequently limited in the content of carbon sources available. To survive in such environments, these pathogens have to control the expression of key metabolic functions. ${ }^{10-12}$ For instance, during gastrointestinal and vaginal colonization, where glucose concentration is low, alternative carbon sources such as acetate or lactate are particularly abundant ${ }^{13,14}$ and may support the growth and the proliferation of $C$. glabrata cells. Interestingly, low-glucose environments were found to induce the formation of C. glabrata biofilms and confer resistance to antifungal treatment. ${ }^{15}$ This behavior suggests that this pathogen has the capacity to adjust its lifestyle in accordance to nutrient availability and determine the outcome of the next phase: either to continue as part of a biofilm population or disperse to find new colonization sites. However, little is known about the physiological effect of acidic environments, containing alternative non-fermentable carbon sources such as acetate, on the antifungal treatment of C. glabrata biofilms. Our group has previously demonstrated that $C$. glabrata cells were more susceptible to fluconazole and better phagocytosed and killed by macrophages, when exposed to both glucose and to physiological concentrations of acetate. ${ }^{16}$ Growth in the presence of this substrate also affected the ability of these cells to form biofilms. ${ }^{16}$ Although several putative acetate transporters and channels were identified to be involved in the response to acetate and fluconazole, in both planktonic and biofilm cells, the exact molecular mechanisms underlying fluconazole resistance in biofilms under acidic conditions are still unclear. In this work, the specific transcriptomic responses of C. glabrata biofilm cells to fluconazole, when grown in the presence of glucose or glucose and acetate, were evaluated by RNA sequencing. This allowed us to decipher the differentially expressed genes and potential mechanisms developed by biofilms to adapt to these different physiological environments.

Our data represent a global view of transcriptomic regulation in C. glabrata biofilms in response to carbon adaptation and fluconazole resistance. Considering the urgent need to find adequate and more effective therapeutic approaches to treat Candida infections, further understanding of the molecular

\footnotetext{
${ }^{1}$ Center of Molecular and Environmental Biology, Department of Biology, University of Minho, Braga, Portugal. ${ }^{2}$ Aberdeen Fungal Group, University of Aberdeen, Institute of Medical Sciences, Foresterhill, Aberdeen, UK. ${ }^{3}$ LIBRO - Laboratório de Investigação em Biofilmes Rosário Oliveira, Center for Biological Engineering, University of Minho, Braga,

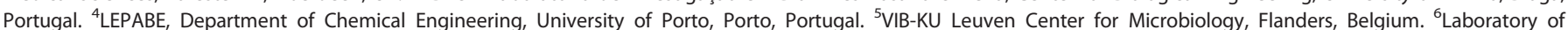
Molecular Cell Biology, Institute of Botany and Microbiology, KU Leuven, Leuven, Belgium. ${ }^{7}$ MRC Center for Medical Mycology, University of Exeter, Geoffrey Pope Building, Stocker Road, Exeter, UK. *email: spaiva@bio.uminho.pt
} 
mechanisms underlying biofilm formation and antifungal resistance could lead to the development of novel inhibitors to control the dissemination of these pathogens.

\section{RESULTS}

Transcriptional responses of $C$. glabrata biofilm cells to fluconazole are modulated by the presence of acetate

C. glabrata biofilms are frequently grown on artificial media conditions that do not mimic physiological environments, leaving the effects of important alternative metabolites largely unexplored. In order to understand the impact of both acidic and acetateenriched environments in response to fluconazole treatment, we evaluated the whole transcriptome of $C$. glabrata biofilm cells by RNA sequencing. Biofilms were developed for $48 \mathrm{~h}$ in RPMI media (with low-glucose concentration, $0.2 \%$ ), either supplemented or not with $0.5 \%$ acetate, and in the presence and absence of fluconazole. This resulted in a total of four different conditions. The total RNA was extracted for 12 independent samples, corresponding to four conditions and three biological replicates. Illumina wholetranscriptome sequencing produced around $1.6 \times 10^{7}\left( \pm 1.4 \times 10^{6}\right)$ reads per sample, which resulted approximately in $98 \%$ overall alignment rate. We then performed pair-wise comparisons between conditions to investigate transcriptional responses to fluconazole treatment, either when biofilms were grown in the presence of acetate (RPMI + acetate) or only in glucose (RPMI).

Figure 1a shows a heatmap depicting the gene regulation of $C$. glabrata biofilms in response to both conditions. The adaptation of biofilm cells to the shift from RPMI to RPMI supplemented with acetate, when treated with fluconazole, was accompanied by extensive changes in gene expression. The data show that the transcriptional response of $C$. glabrata biofilm cells to fluconazole is clearly modulated by the presence of acetate (Fig. 1). For both comparisons, we obtained lists of genes significantly up- and downregulated and analyzed those displaying at least twofold regulation (Fig. 1b). We also performed a global functional analysis on the identified genes in order to find significantly enriched GO biological process terms and better understand the biological mechanisms associated with them.
The transcription of genes involved in DNA replication, ergosterol, and ubiquinone biosynthesis is modulated by the presence of acetate in response to fluconazole treatment

In order to characterize the transcriptional responses to fluconazole of $C$. glabrata biofilms grown in the presence of acetate, we compared the transcript profiles of samples grown in RPMI media supplemented with acetate and fluconazole, with those grown just in RPMI supplemented with acetate. Upregulated genes were significantly enriched in biological categories such as "DNA replication initiation" (CgMCM6 and CgMCM7), "ergosterol biosynthetic process" (CgERG9 and CgERG11) and "ubiquinone biosynthetic process" (CgCAT5, CgCOQ6, and CgCOQ9; Fig. 2). The overexpression of both Mcm6p and Mcm7p, which are associated with the MCM complex that initiates and regulates DNA replication, ${ }^{17}$ may be indicative of increased cell proliferation that ultimately may lead to chromosomal instability. This hypothesis is in agreement with previous observations correlating chromosomal abnormalities in several Candida spp. with exposure to fluconazole. ${ }^{18}$ However, this response has never been associated with the presence of acetate or other non-fermentable carbon sources during C. glabrata biofilm growth. Considering the overexpression of ergosterol biosynthetic genes, this is one of the most common mechanisms of resistance in response to fluconazole treatment in Candida spp. ${ }^{18}$ The mechanism of action of fluconazole involves the inhibition of cytochrome P450 enzyme lanosterol demethylase, encoded by ERG 11. ${ }^{19}$ This reaction occurs due to the binding of a free nitrogen atom of the azole ring to an iron atom within the heme group of the enzyme. This binding prevents oxygen activation and consequently demethylation of lanosterol, resulting in the inhibition of ergosterol biosynthesis. Moreover, Erg9p is also involved in ergosterol biosynthesis but functions upstream of lanosterol demethylase in the biosynthetic pathway. ${ }^{20}$ As ergosterol is an important component of fungal cell membranes, any impairment in the ergosterol biosynthetic pathway results in increased cellular permeability leading to the disruption of cell membranes. Thus, ergosterol overexpression confers protection against fluconazole treatment by maintaining sterol homeostasis. Lastly, the three genes encoding proteins involved in the ubiquinone biosynthesis, Coq6p, Cat5p and Coq9p, are part of a multi-subunit complex of nine proteins

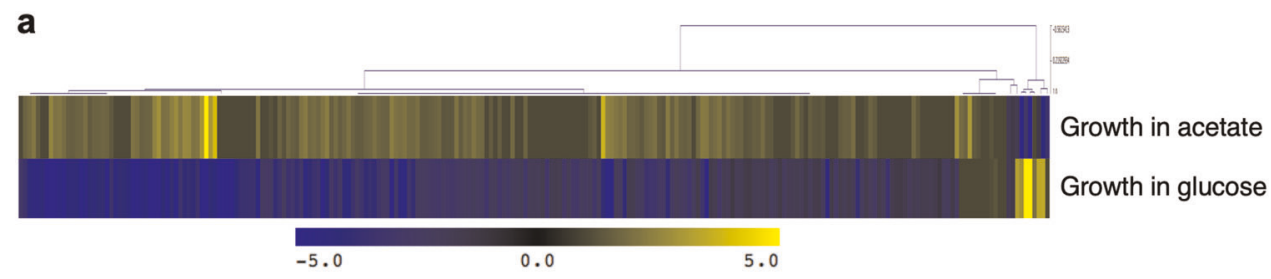

b

Downregulated genes

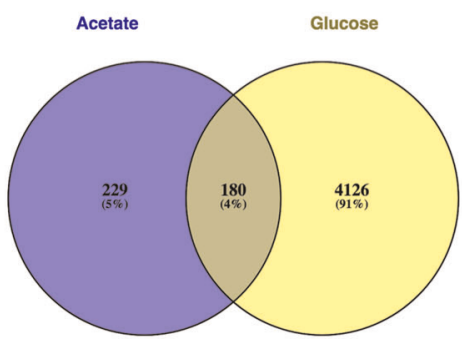

Upregulated genes

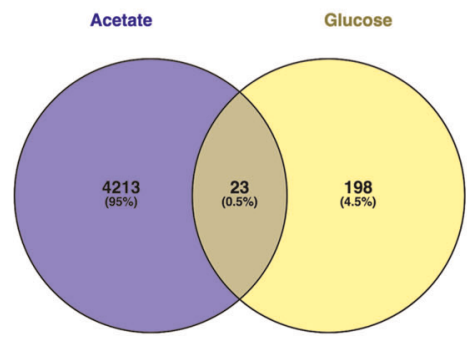

Fig. 1 Global transcriptional response of C. glabrata biofilm cells to fluconazole treatment when grown in the presence of glucose (RPMI + fluconazole versus RPMI) or in the presence of both glucose and acetate (RPMI + acetate + fluconazole versus RPMI + acetate). a Heatmap of all genes differentially expressed $(p<0.05)$ in the presence versus the absence of fluconazole in both growth conditions. $\mathbf{b}$ Venn diagrams of downregulated (decrease of twofold or lower) and upregulated (greater than twofold) genes in C. glabrata biofilm cells due to fluconazole treatment in both growth conditions. 
Downregulation

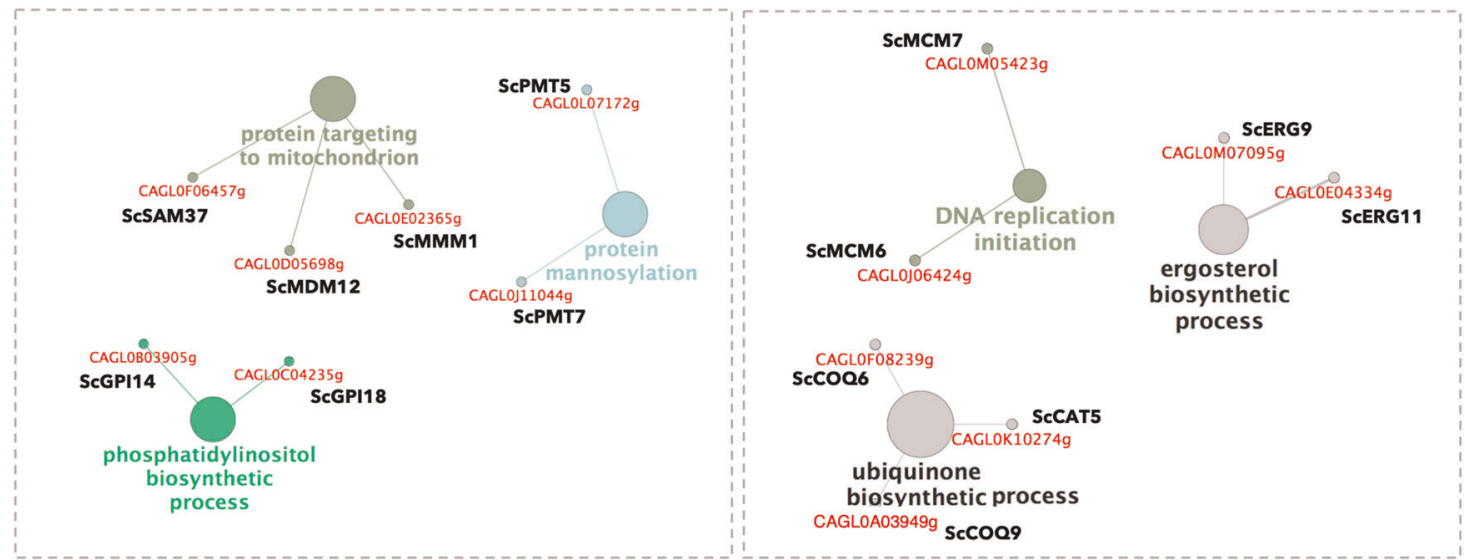

Fig. 2 Transcriptional response of C. glabrata biofilm cells to fluconazole when grown in the presence of acetate. Network visualization of enriched pathways with the systematic names of C. glabrata (downregulated, left panel; and upregulated, right panel) when grown in the presence of acetate and fluconazole was performed by ClueGO analysis. The size of the nodes represents the statistical significance of the terms. The systematic names of $C$. glabrata genes and respective orthologs in $S$. cerevisiae associated with each biological process are shown in red and black, respectively.

Downregulation

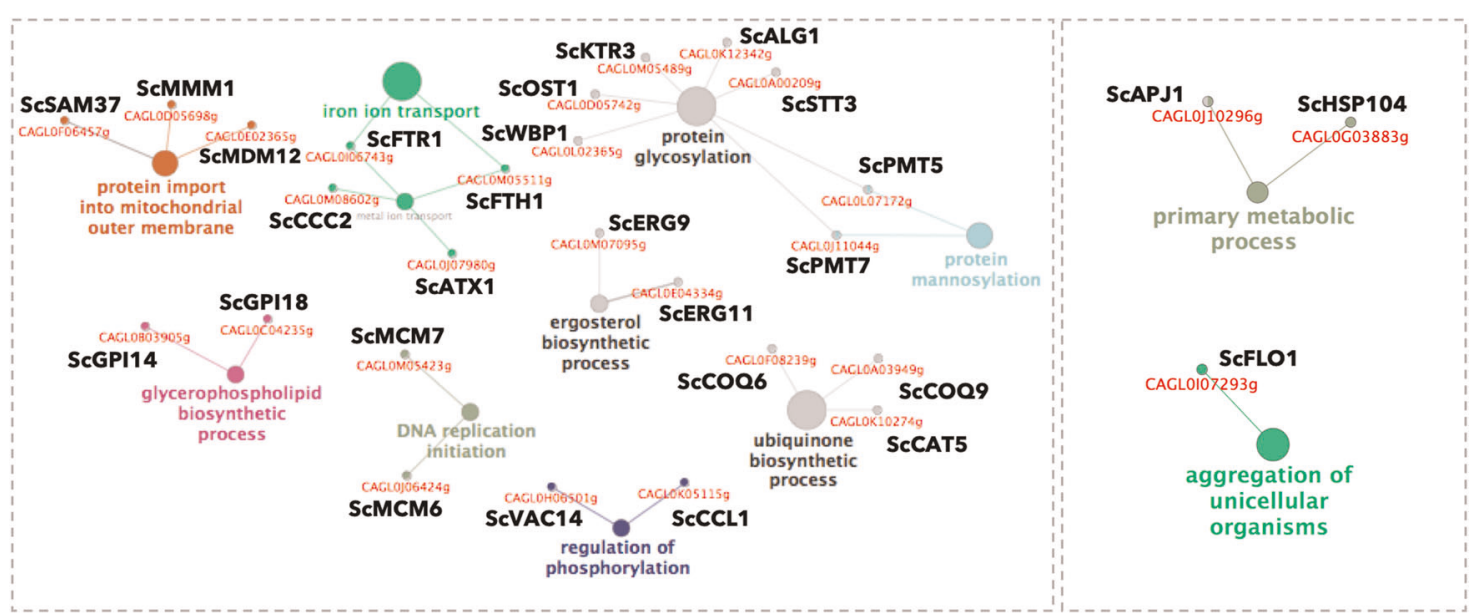

Fig. 3 Transcriptional response of C. glabrata biofilm cells to fluconazole, grown in the presence of glucose, as sole carbon source. Network visualization of enriched pathways with the systematic names of C. glabrata (downregulated, left panel; and upregulated, right panel) when grown in the presence of glucose and fluconazole was performed by ClueGO analysis. The size of the nodes represents the statistical significance of the terms. The systematic names of C. glabrata genes and respective orthologs in S. cerevisiae associated with each biological process are shown in red and black, respectively.

required for ubiquinone biosynthesis. ${ }^{21-24}$ Ubiquinone, also known as Coenzyme $Q$, is an essential component of the electron transport chain, carrying electrons from Complexes I and II to Complex III in mitochondria. These proteins are required for respiratory growth and gluconeogenic gene activation. ${ }^{21-23}$ Indeed, during growth on non-fermentable carbon sources, such as acetate, Candida cells induce gluconeogenesis in order to obtain sugar phosphates for the synthesis of essential cellular components. ${ }^{25}$ Collectively, these data suggest that fluconazole increases chromosome instability, ergosterol biosynthesis and endogenous respiration in C. glabrata biofilm cells grown in the presence of acetate (Fig. 2). When grown in the presence of glucose as sole carbon source, all of these genes were found to be downregulated (Fig. 3), suggesting that these pathways are regulated in a carbon source-dependent manner.
Reduction of mitochondrial activity appears to be induced in response to fluconazole treatment in a manner independent of carbon source

On the other hand, downregulated genes were significantly enriched in biological processes related to alterations in mitochondrial biogenesis and cell wall organization, as a response to fluconazole treatment. Genes involved in "protein targeting to mitochondrion" (CgSAM37, CgMDM12, and CgMMM1) codify a set of translocases that are part of a Sorting Assembly Machinery (SAM) complex that mediates the insertion of beta-barrel proteins into the mitochondrial outer membrane. ${ }^{26-28}$ The core of this complex is composed of Sam50p, Sam37p and Sam35p, ${ }^{29-32}$ but other proteins such as $M d m 12 p$ and $M m m 1 p$ are required to successfully complete this process. ${ }^{33}$ This transcriptional response is in accordance with previous transcriptomics and metabolomics 
analysis in C. albicans that suggest a downregulation of mitochondrial activity as a result of biofilm maturation. ${ }^{34-37}$ Other studies have also suggested that the reduction of mitochondrial activity might be beneficial for biofilm growth, conferring protection against oxidative damage and promoting prolonged survival of biofilm cells. ${ }^{38,39}$ The downregulation of these genes provides additional evidence that the loss of mitochondrial functions is associated with fluconazole resistance in C. glabrata. Other biological categories also found to be downregulated due to fluconazole treatment include "protein mannosylation" (CgPMT5 and CgPMT7) and "phosphatidylinositol biosynthetic process" (CgGPI14 and CgGPI18; Fig. 2). The cell wall glycoproteins of Candida spp. are modified by $\mathrm{N}$ - and $\mathrm{O}$-linked glycosylation and are attached to the polysaccharides at the cell wall by either covalent links or glycosylphosphatidylinositol (GPI) anchors. ${ }^{40}$ The protein O-mannosyltransferases (PMTs) are an evolutionarily conserved, essential family of proteins that mannosylate distinct targets in the endoplasmic reticulum. ${ }^{41}$ In C. albicans, where these proteins have been well studied, this posttranslational modification was shown to be involved in morphogenesis, adhesion and antifungal resistance, suggesting that PMTs can be studied as potential antifungal targets. ${ }^{42-44}$ Both Gpi14p and Gpi18p are also mannosyltransferases but specifically responsible for adding $a-1,4$-linked and $a-1,6$-linked mannoses to GPI, respectively. ${ }^{45-47}$ As in fungi all of these genes have several subfamily members that are highly redundant, the effect of their downregulation is unclear. Interestingly, our data show that upon fluconazole treatment, biofilm cells grown only in the presence of glucose exhibit the same transcriptional pattern of regulation for all categories mentioned above (Figs 2 and 3). We conclude that these transcriptional responses to fluconazole are independent of the carbon source.

In glucose-grown biofilm cells, fluconazole treatment induces the overexpression of genes involved in metabolism, protein folding, and adhesion

To characterize the transcriptional response of glucose-grown biofilm cells to fluconazole, we compared the transcript profile of samples grown in RPMI and fluconazole with those grown in RPMI. Upregulated genes due to fluconazole treatment were significantly enriched in processes related to "primary metabolic process" (CgAPJ1 and CgHSP104) and aggregation (CgFLO1, also known as CgEPA1) (Fig. 3). Apj1p is a J protein cochaperone of the HSP40 family while Hsp104p is a general anti-stress chaperone that in conjunction with other proteins helps to disassemble protein aggregates accumulated due to stress. ${ }^{48,49}$ Heat-shock proteins (HSPs) are induced by several types of stress and have broadly protective functions. ${ }^{50}$ Their expression has been associated with biofilm formation and drug resistance in Candida spp., reinforcing their potential as antifungal targets. ${ }^{48,50,51}$ Some links between azole resistance and protein folding functions have also been reported in C. albicans. ${ }^{52,53}$ CgEPA1 is a GPI-anchored cell wall protein that participates in cellcell interactions, allowing adherence to host epithelial tissues. ${ }^{54}$ In C. glabrata, Epa1p is the major adhesin mediating adhesion and its overexpression is associated with resistance to fluconazole. ${ }^{55}$

Iron acquisition and glycosylation appear to be attenuated by fluconazole treatment when glucose is present, as sole carbon source

The transcriptional responses to fluconazole when C. glabrata biofilm cells were grown in the presence of glucose are associated with a higher number of differentially regulated genes when compared with the acetate-dependent responses described above. Downregulated genes were significantly enriched in several processes (Fig. 3), including "iron ion transport" (CgFTR1, CgFTH1, CgCCC2 and CgATX1). Since Ftr1p, Fth1p and Ccc2p are part of the high-affinity iron uptake system in C. glabrata, and Atx1p is also involved in iron absorption, downregulation of these genes may result in perturbed iron homeostasis. ${ }^{56}$ Given that iron acts as a cofactor for numerous metalloproteins involved in several fundamental cellular processes, such as oxygen transport, respiration, energy metabolism and DNA synthesis and repair, perturbations in iron homeostasis also have a direct impact on these processes. This is consistent with some of the downregulated categories found under the same conditions, namely, ergosterol biosynthesis. Additionally, genes involved in "protein glycosylation" (CgWBP1, CgOST1, CgKTR3, CgALG1 and CgSTT3) were also found to be downregulated in response to fluconazole (Fig. 2). The cell wall glycoproteins in Candida spp. can be $\mathrm{N}$ - or $\mathrm{O}$-glycosylated. The $\mathrm{N}$-linked glycosylation pathway requires the action of different glycosyltransferases and comprises two sequential stages. ${ }^{40,57}$ The first stage involves the synthesis of a dolichol-linked glycan precursor and its transfer to a nascent protein in the rough endoplasmic reticulum. Alg1p is involved in the synthesis process and Wbp $1 \mathrm{p}, \mathrm{Stt} 3 \mathrm{p}$, and Ost1p are transmembrane subunits of the oligosaccharyl transferase complex (OST) that mediate the transference. ${ }^{57}$ The second stage includes the $\mathrm{N}$-linked glycan processing and maturation in the rough endoplasmic reticulum and Golgi. CgKTR3, a member of the $K R E 2 / M N T 1$ gene family, may be involved in this last stage but also in $\mathrm{O}$-linked protein glycosylation. ${ }^{58} \mathrm{~A}$ reduction in the levels of glycosylation will impact the composition of the cell wall and thus the interaction between the biofilm cells. This is consistent with data from $C$. albicans showing that changes in carbon source affect cell wall architecture, the cell wall proteome and cell wall organization. ${ }^{59-61}$ Besides glycosylation, some proteins involved in the "regulation of phosphorylation" (CgCCL1 and CgVAC14) also appear to be downregulated in response to fluconazole treatment (Fig. 3). CgCCL1 positively regulates transcription by Polymerase II by stimulating phosphorylation of RNA polymerase II C-terminal domain, with mutants showing increased competitive fitness in Saccharomyces cerevisiae. ${ }^{62}$ Vac14p controls the synthesis of phosphatidylinositol-3,5-biphosphates, impacting several mechanisms such as organelle morphology, membrane recycling, and ion transport. $^{63}$ Altogether, these biological categories represent a deregulation in mitochondrion function, cell cycle, cell wall composition, iron transport, and post-translation modifications, such as mannosylation, glycosylation, and phosphorylation.

\section{Validation of RNA-seq results with quantitative real-time PCR} (qRT-PCR)

In order to validate the RNA-seq findings, qRT-PCR was performed on all conditions for six genes that are key members of the most significant differentially expressed categories found by $\mathrm{GO}$ analysis: CAT5, COQ6, ERG9, ERG11, FTR1, and ATX1 (Fig. 4). All of these genes were also tested when $C$. glabrata biofilm cells were grown in the presence of fluconazole, and when using glucose as sole carbon source at $\mathrm{pH}$ 5.0, to check whether the $\mathrm{pH}$ influenced the changes in gene expression (Supplementary Fig. 1). Significantly, the changes in expression observed by RNA-seq were confirmed by qRT-PCR for all the tested genes. Furthermore, the changes in expression observed between growth conditions were independent of the pH (Fig. 4 and Supplementary Fig. 1).

CgMge1 drives fluconazole resistance in acidic and acetateenriched environments

C. glabrata is able to colonize and infect acidic and acetateenriched anatomical sites, including the gastrointestinal tract and vagina. We identified a set of genes that are differently regulated due to fluconazole treatment, when cells are growing in conditions mimicking those acidic environments. Considering the overexpression of both CgERG9 and CgERG11 in response to fluconazole, when Candida biofilm cells are growing in the 
CAT5

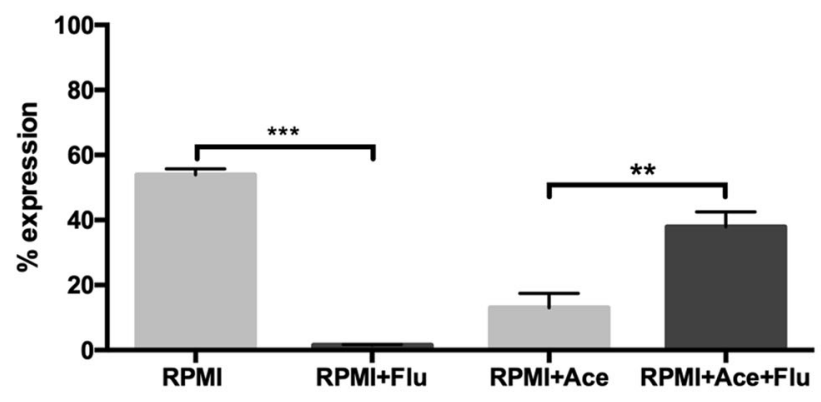

ERG9

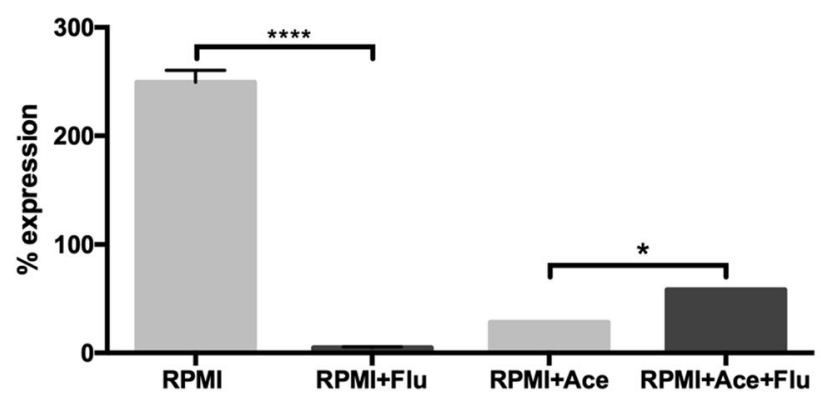

FTR1

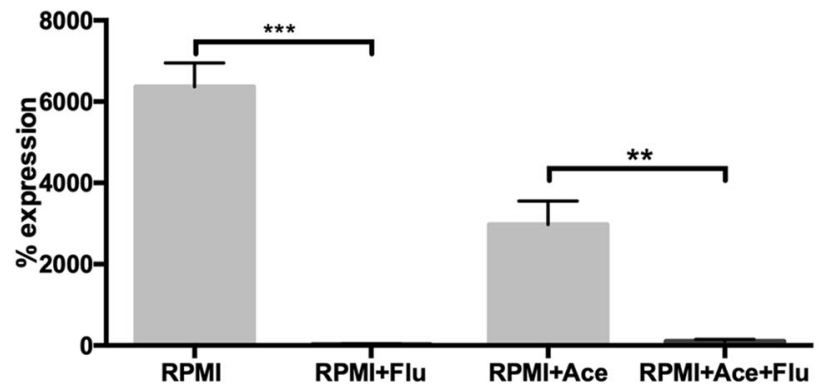

COQ6

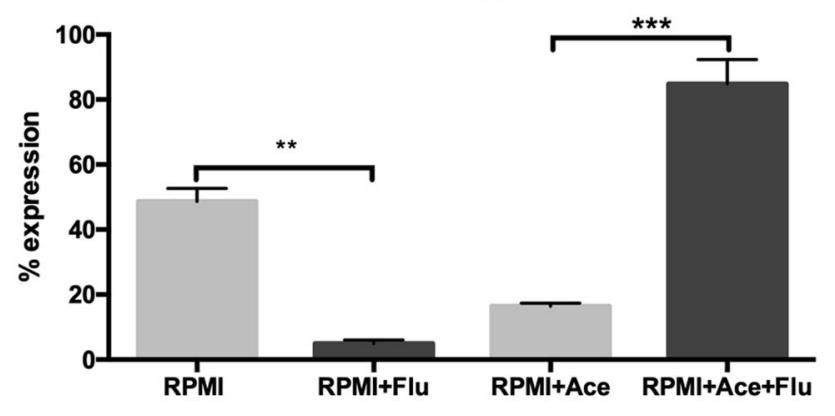

ERG11

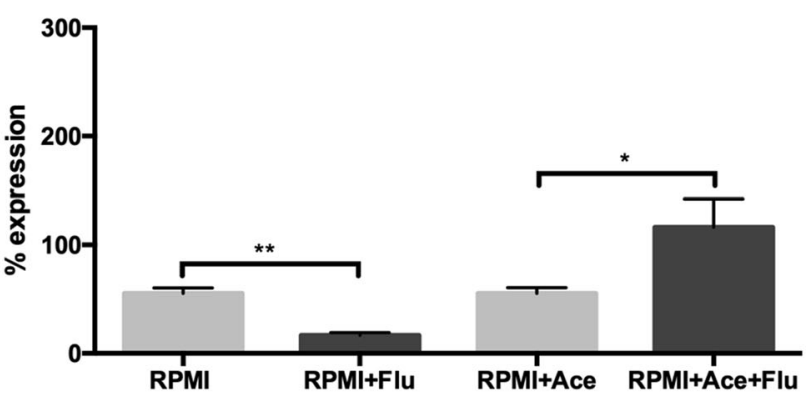

ATX1

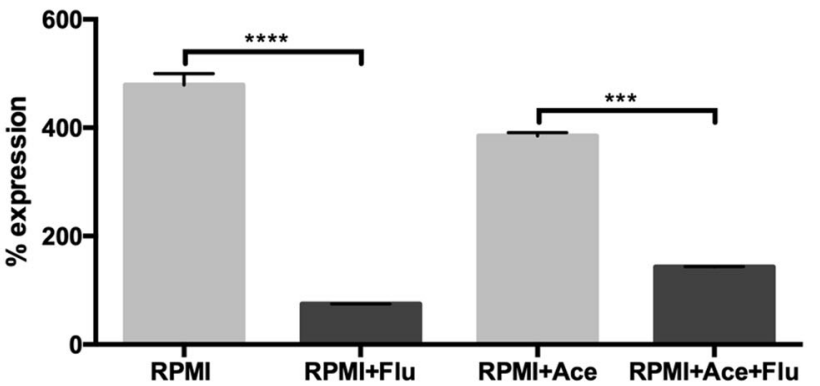

Fig. 4 Validation by qRT-PCR of genes that were differentially expressed (CAT5, COQ6, ERG9, ERG11, FTR1, and ATX1) in response to fluconazole in C. glabrata biofilm cells grown with glucose as sole carbon source (RPMI; pH 7.0) or supplemented with acetate (RPMI + Ace; pH 5.0). Graphs show percentage expression of each gene compared to a housekeeping gene, PGK1. The error bars show standard deviation. ${ }^{*} p<0.05,{ }^{* *} p<0.01,{ }^{* * *} p<0.001,{ }^{* * *} p<0.0001$ were considered statistically significant relative to untreated $C$. glabrata biofilm cells.

presence of acetate (Fig. 2), we postulated that C. glabrata would display increased resistance to fluconazole under those conditions. To physiologically validate our transcriptional data, $C$. glabrata cells were grown in the presence of fluconazole and/or doxycycline, an iron chelator reported to have a synergistic effect with fluconazole. ${ }^{64-66}$ As a control strain, we used an overexpression mutant for MGE1 driven by the CgTDH3 promoter in the $2001 \mathrm{HTL}$ strain (Fig. 5). ${ }^{65}$ This gene encodes a putative mitochondrial matrix cochaperone, which is a known suppressor of fluconazole susceptibility in both C. albicans and C. glabrata. ${ }^{65}$ Since CgMGE1 is upregulated when cells are treated with fluconazole, under acidic environments (Fig. 5a), we hypothesized that WT cells would display the same phenotype as MGE1overexpressing cells, when grown under similar conditions. We tested this, and all experiments were performed in triplicate, showing consistent results among all the independent assays. Growth conditions were maintained by supplementing RPMI medium containing $0.2 \%$ glucose with the respective stressor and adjusting the $\mathrm{pH}$ to 7.0 or 5.0. This enabled us to compare our physiological data with the transcriptional outputs.
Phenotypes from control conditions (RPMI pH 7.0) for the MGE1overexpressing strain were in agreement with previously published data, where CgMGE1 overexpression was shown to play a role in regulating susceptibility to fluconazole (Fig. 5b). ${ }^{65}$ As expected, cells grown under acidic conditions, such as RPMI at pH 5.0 and RPMI at pH 5.0 supplemented with $0.5 \%$ acetate, were more resistant to fluconazole and doxycycline than those grown only in RPMI at pH 7.0 (Fig. 5b). Interestingly, the synergistic effect of doxycycline was strongly modulated by $\mathrm{pH}$ and the carbon source, since this effect was abolished under acidic $\mathrm{pH}$, and moderately restored for the highest concentration of fluconazole in the presence of acetate (Fig. 5b). As we predicted, both WT and MGE1-overexpressing cells displayed the same phenotype under acidic conditions (Fig. 5b). We can conclude from these data that overexpression of MGE1 under acidic conditions (confirmed both by RNA-seq and RT-PCR; Fig. 5a) causes a decrease in the susceptibility of $C$. glabrata to fluconazole (Fig. 5b). Our data also highlight the importance of mimicking distinct environmental niches as local inputs significantly affect the responses of Candida cells to antifungal treatment. 
MGE1 expression

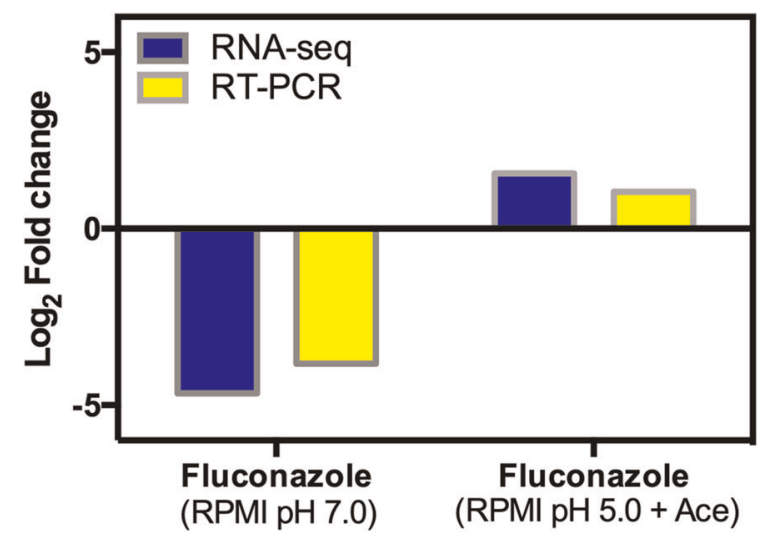

\section{Growth Condition}

b

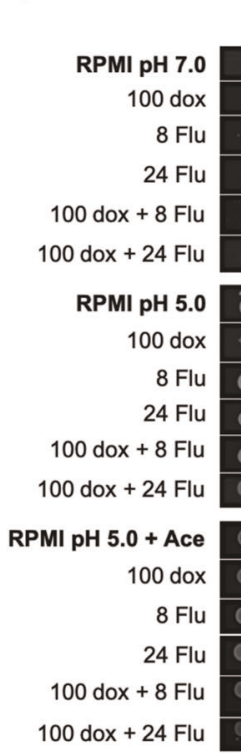

$48 \mathrm{~h}$

WT
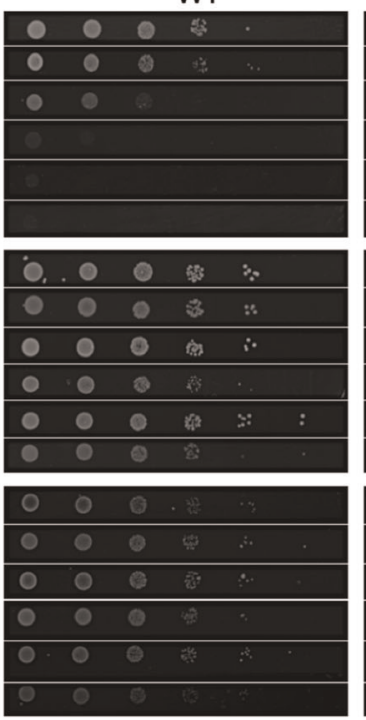

MGE1
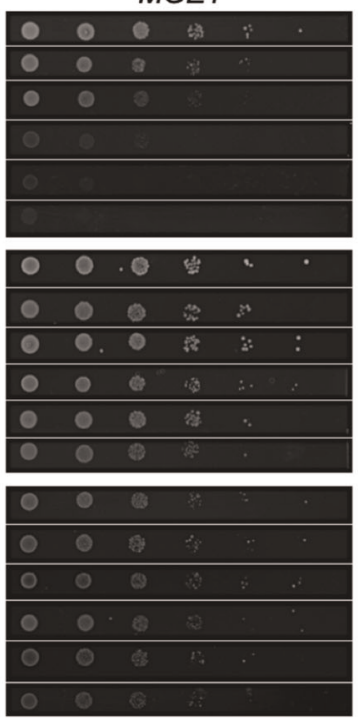

$72 \mathrm{~h}$
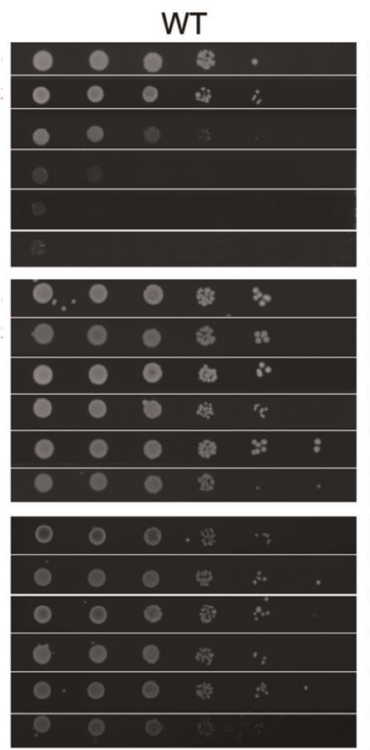

MGE1
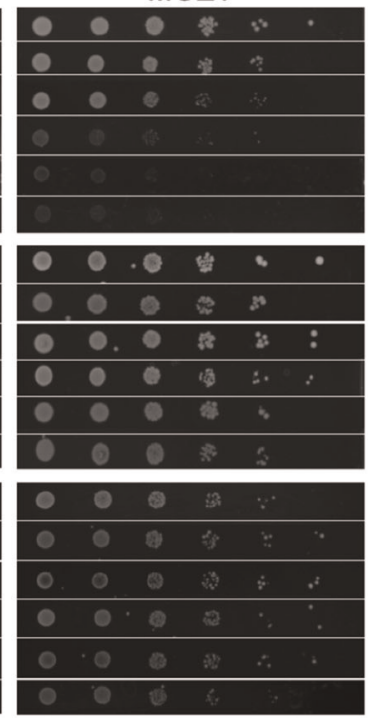

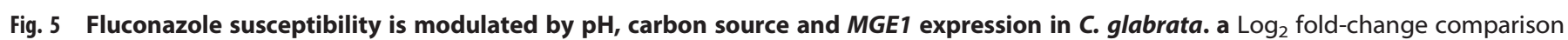
of RNA-seq (blue bars) and quantitative RT-PCR (yellow bars) for MGE1 expression in response to fluconazole treatment when C. glabrata cells are growing either using glucose as sole carbon source (RPMI pH 7.0) or in the presence of acetate (RPMI pH $5.0+$ Ace). b Serial dilutions of wild-type strain (WT; 2001HTL strain transformed with the empty plasmid as a control) and overexpression strain (MGE1; 2001HTL strain transformed with a plasmid expressing CgMGE1 ORF, together with its terminator, from the CgTDH3 promoter ${ }^{65}$ ) were spotted on RPMI medium containing $0.2 \%$ glucose at $\mathrm{pH} 7.0 \mathrm{or} \mathrm{pH} 5.0$, and/or containing acetate (ace; $0.5 \% \mathrm{v} / \mathrm{v})$, and/or fluconazole (flu; $8 \mathrm{or} 24 \mu \mathrm{gg} / \mathrm{mL}$ ) and/or doxycycline (dox; $100 \mu \mathrm{g} / \mathrm{mL}$ ). Pictures were taken after $48 \mathrm{~h}$ and $72 \mathrm{~h}$ of incubation at $37^{\circ} \mathrm{C}$.

Overexpression of CgMGE1 reduces toxic sterol formation

Ergosterol biosynthesis is inhibited in the presence of fluconazole, favouring the formation of toxic sterols due to the accumulation of lanosterol (Fig. 7a). Previous work has demonstrated that the overexpression of CgMGE1 reduces the metabolic flux to toxic sterol formation. ${ }^{65}$ Therefore, we performed gas chromatographymass spectrometry (GC-MS) analysis to investigate the possible role of sterols in mediating fluconazole resistance, which is higher when C. glabrata cells grow in acidic environments and when MGE1 is upregulated. Sterols were isolated from both WT and MGE1-overexpressing cells grown in RPMl at $\mathrm{pH} 7.0$, RPMI at $\mathrm{pH} 5.0$ and $\mathrm{RPMI}$ at $\mathrm{pH} 5.0+0.5 \%$ acetate, in the presence and absence of fluconazole (Fig. 6b). In addition to sterols such as ergosterol and lanosterol, we detected other sterols that were less abundant or could not be identified (Supplementary Fig. 2). This approach was validated by using an alternative sterol quantitation method (SQM; Supplementary Fig. 3). ${ }^{67}$

As can be seen from Fig. $6 \mathrm{~b}$, fluconazole treatment leads to lanosterol accumulation and ergosterol depletion under all the growth conditions examined. This accumulation favors the formation of toxic sterols such as 14-methylergosta-8,24(28)dien-3ß,6a-diol. Remarkably, MGE1-overexpressing cells displayed reduced levels of this toxic sterol (Fig. 6b). This reduction is statistically significant only for cells grown under acidic conditions $(p<0.001$ and $p<0.05$ for RPMI $\mathrm{pH} 5.0$ and RPMI $\mathrm{pH} 5.0+0.5 \%$ acetate, respectively). Additionally, the balance between ergosterol and 14-methylergosta-8,24(28)-dien-3 $3,6 \mathrm{a}$-diol in these cells is higher for RPMI at pH 5.0 and RPMI at pH $5.0+0.5 \%$ acetate (Fig. 6b). By reducing the metabolic flux towards 14-methylergosta-8,24(28)-dien-3ß,6a-diol, other sterol intermediates can 
a

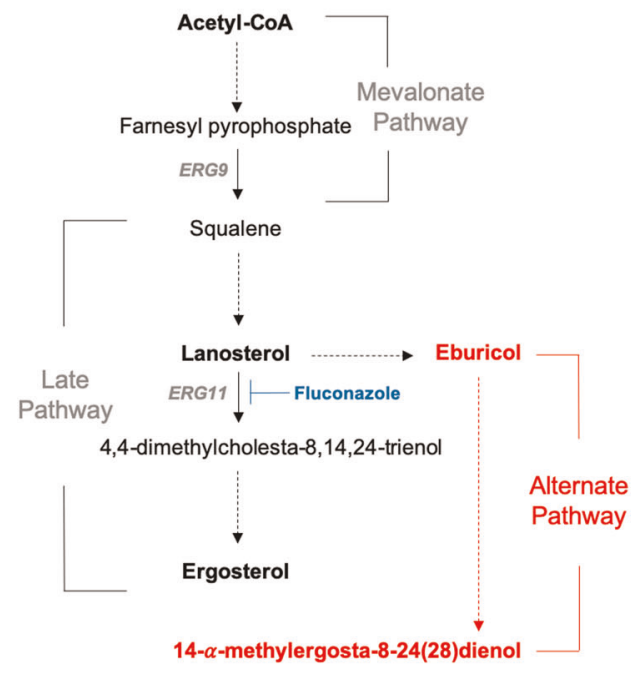

b
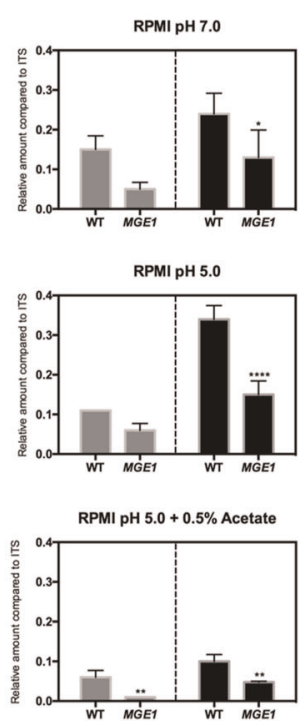

Ergosterol
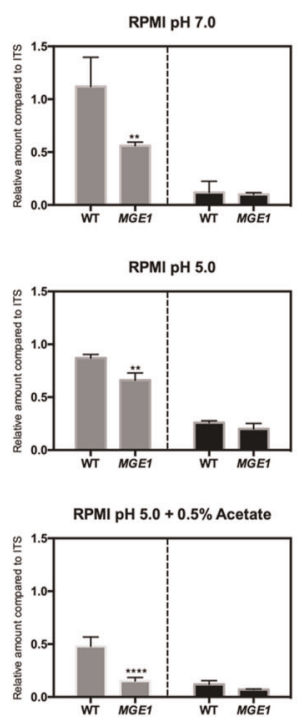

14- $\alpha$-methylergosta-8-24(28)dienol
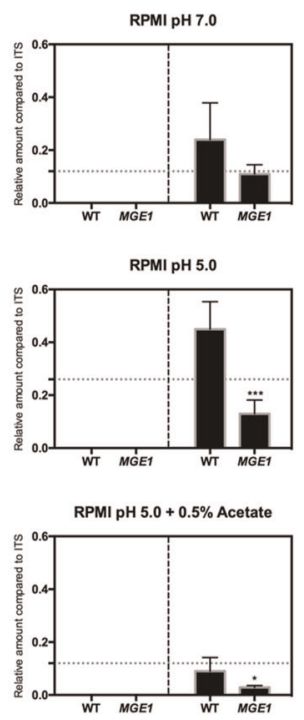

no fluconazole

Fig. 6 MGE1 overexpression modulates the aberrant flux of sterols following fluconazole treatment. a Schematic representation of sterol biosynthetic pathways. Erg11 is the primary target of fluconazole. Red names represent toxic fungistatic sterols. b CgMGE1 overexpression reduces toxic sterol formation. Cells were grown in RPMI medium containing $0.2 \%$ glucose at $\mathrm{pH} 7.0$, or $\mathrm{pH} 5.0$, and/or containing $0.5 \%$ acetate, in the presence or absence of fluconazole for $24 \mathrm{~h}$. Sterol levels were determined by GC-MS and are displayed for lanosterol, ergosterol, and 14-methylergosta-8,24(28)-dien-3 $3,6 \alpha$-diol. The values were calculated relative to the internal standard (ITS; cholestane). The error bars show standard error of the mean. ${ }^{*} p<0.05,{ }^{* *} p<0.01,{ }^{* * *} p<0.001,{ }^{* * *} p<0.0001$ were considered statistically significant relative to C. glabrata WT cells. Gray dotted grid lines in each condition represent the mean obtained for ergosterol content for the same MGE1overexpressing cells.

migrate to the plasma membrane and replace the essential ergosterol functions. Considering that under these conditions, $C$. glabrata cells present higher fluconazole resistance (Fig. 5b), these data present additional evidence for the role of MGE1 in reducing the levels of toxic sterols and, consequently, decreasing susceptibility to fluconazole.

\section{DISCUSSION}

Natural niches of C. glabrata include the human gastrointestinal and vaginal tracts, ${ }^{68}$ environments particularly rich in alternative carbon sources and often limited in the amount of glucose. ${ }^{11}$ In order to thrive in these changing nutrient environments, $C$. glabrata has evolved sophisticated regulatory mechanisms, including major metabolic changes that have been associated with virulence and, in particular, biofilm formation. ${ }^{15,16,69}$ Once established, Candida biofilms are very difficult to eradicate due to their intrinsic tolerance to antifungals. In fact, azole resistance among Candida species, especially C. glabrata, is currently one of the greatest clinical challenges. ${ }^{2,4,7}$ Here, we investigated the effect of different carbon sources on fluconazole-treated $C$. glabrata biofilms by using a RNA-seq approach. For that, biofilms were grown in the presence of low glucose or low glucose plus acetate, a non-fermentable carbon source frequently found in several host niches. ${ }^{14,70}$ The differentially regulated genes identified in both growth conditions allowed us to decipher the potential molecular mechanisms associated with carbon adaptation and fluconazole resistance. Indeed, since most of the genes are still uncharacterized in C. glabrata, their function was predicted based on their orthologs in the phylogenetically closely related yeast $S$. cerevisiae.

Depending on the growth condition, some genes were found to be differently regulated in response to fluconazole. These included genes encoding proteins involved in DNA replication, mitochondrial function, and ergosterol biosynthesis (Figs 2 and 3). While this observation could be anticipated for the genes involved in DNA replication and mitochondrial function, as metabolism is finely coordinated with cell growth, chromosome replication and cell division, it was quite surprising to find that the carbon source affects the degree to which fluconazole induces ergosterol-related genes. As sterol biosynthesis requires heme, we hypothesize that the expression of these genes is also dependent on the status of iron acquisition in the cells. Indeed, the expression profile of iron-related genes is finely tuned with those involved in the expression of ergosterol (Figs 2-4). Curiously, even with all of these impairments, C. glabrata cells from biofilms still survive under high concentrations of fluconazole. ${ }^{16}$ In fact, it was already shown that deprivation of iron does not seem to affect the ability of cells to form biofilms in C. albicans. ${ }^{71}$ Moreover, the lack of iron enhances membrane fluidity and drug diffusion in C. glabrata planktonic cells, ${ }^{66}$ consistent with the impairment in ergosterol biosynthesis. On the other hand, our analyses provided additional evidence that the loss of mitochondrial function is associated with fluconazole resistance, independently of the growth condition. Additionally, while physiologically validating our transcriptional outputs, we realized that C. glabrata Mge1, a cochaperone involved in iron metabolism and protein import into the mitochondria, might influence fluconazole susceptibility during carbon and $\mathrm{pH}$ adaptation. We confirmed this experimentally (Fig. 6), thereby reinforcing the role of CgMGE1 in mediating fluconazole resistance by reducing toxic sterol formation. ${ }^{65}$

In this study, we did not observe statistically significant deregulations regarding several well-known genes involved in fluconazole resistance in planktonic C. glabrata cells. These include the induction of efflux pumps, encoded by the $A B C$-transporter genes, such as CgCDR1, CgCDR2 (also known as CgPDH1), and CgSNQ2, which are regulated mainly by the transcription factor CgPDR 1. ${ }^{2}$ Our data suggest that their expression levels differ 
a
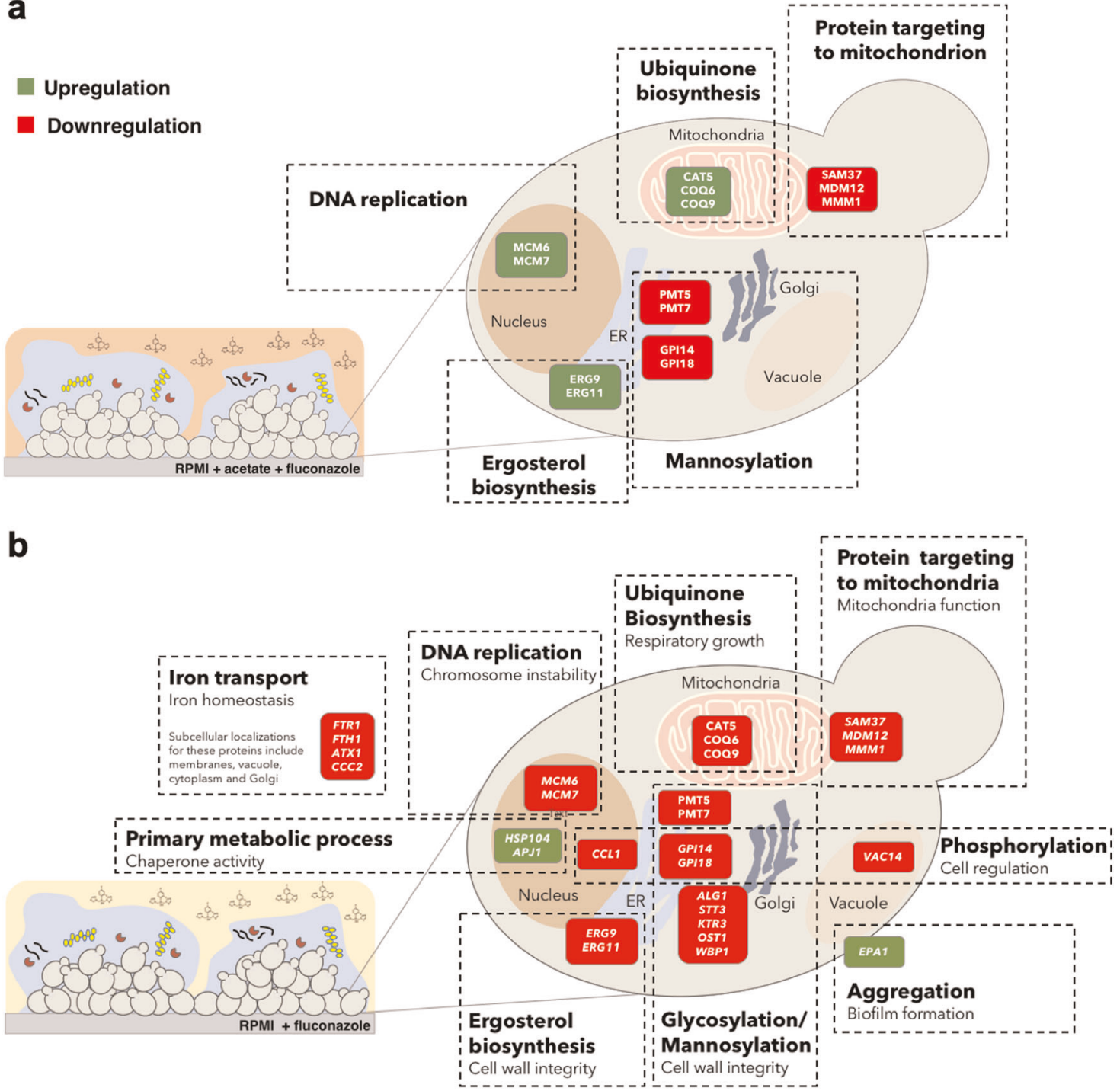

Fig. 7 Schematic overview of the transcriptional responses of $\boldsymbol{C}$. glabrata biofilms to fluconazole. Differentially expressed genes upon fluconazole treatment when cells are grown in the presence of a glucose and acetate or $\mathbf{b}$ glucose.

markedly between biofilms and planktonic cells (Supplementary Fig. 4). This observation is consistent with previous work done in C. albicans. $^{72}$ Mutants knocked out in these genes were hypersusceptible to fluconazole when growing as planktonic cells, but not when growing as biofilms. ${ }^{72}$ This may reflect altered responses due to the characteristics intrinsically related with the biofilm lifestyle, such as growth on a surface, the presence of a protective extracellular matrix, differences in nutrient requirements and metabolism, and/or quorum-sensing mechanisms. ${ }^{73}$

Based on the data presented here, we propose a model in which resistance to fluconazole in C. glabrata biofilms is modulated by the available carbon source and $\mathrm{pH}$ (Fig. 7). This is particularly relevant for a set of genes involved in DNA replication, ergosterol, and ubiquinone biosynthesis. When grown in the presence of glucose, responses to fluconazole treatment are extensive and involve the deregulation of several cellular functions (Fig. 7a). On the other hand, when acetate is present in the growth media, fluconazole treatment impacts mainly chromosome instability, cell wall integrity, and mitochondrial function (Fig. 7b).

Our analyses provide a global overview of the genetic and regulatory mechanisms developed by this microorganism in response to antifungal treatment, and highlights the impact of acidic environments on these processes. These alterations at transcriptional level following fluconazole treatment, either in the presence of glucose or acetate, enhance the robustness of $C$. glabrata biofilms to tolerate this antifungal. Our data reveal new roles of uncharacterized C. glabrata genes and provide more knowledge towards the identification of new potential antifungal targets. Understanding the mechanisms behind fluconazole resistance may improve and facilitate the development of new antifungal compounds to treat Candida infections.

\section{METHODS}

Media, strains, and growth conditions

Biofilm experiments were done using Candida glabrata ATCC 2001. This strain was subcultured on Sabouraud dextrose agar (SDA, Difco, Le Pont de Claix, France) medium at $37^{\circ} \mathrm{C}$ for $24 \mathrm{~h}$ and then inoculated in Sabouraud dextrose broth (SDB; Difco) medium at $37^{\circ} \mathrm{C}$ for $20-24 \mathrm{~h}$ while shaking at $120 \mathrm{rpm}$. Yeast cells were harvested by centrifugation after the incubation period $\left(6500 \times g\right.$, for $10 \mathrm{~min}$ at $\left.4^{\circ} \mathrm{C}\right)$, washed twice in phosphate buffered saline (PBS; pH 7.0, $0.1 \mathrm{M}$ ), and the cellular concentration was adjusted to $1 \times$ $10^{7}$ cells $/ \mathrm{mL}$ using an improved Neubauer chamber. All experimental assays were carried out in filter-sterilized RPMI 1640 medium with L-glutamine (Sigma, R6504) and buffered with $0.165 \mathrm{M}$ morpholinepropanesulfonic acid at 
pH 7.0 or 5.0. Fluconazole (Sigma; F8929), doxycycline (Sigma, D9891) and acetic acid (Sigma, A6283) were added to the medium at concentrations of 8 or $24 \mu \mathrm{g} / \mathrm{mL}$, $100 \mu \mathrm{g} / \mathrm{mL}$, and $0.5 \%(\mathrm{v} / \mathrm{v})$, respectively (unless otherwise stated). Depending on the assay, autoclave-sterilized and precooled agar was added to the medium. Cell cultures containing fluconazole or doxycycline were always kept in the dark. Detailed information about MGE1-overexpressing strain and respective wild-type control can be found in Demuyser et ll. $^{65}$

\section{Biofilm development}

Candida biofilms were developed on 24-well polystyrene plates (Orange Scientific, Braine-l'Alleud, Belgium; 12 wells for each condition) containing $1 \mathrm{~mL}$ of $C$. glabrata cell suspension $\left(1 \times 10^{7}\right.$ cells $/ \mathrm{mL}$ in RPMI 1640$)$ per well. The plates were then incubated at $37{ }^{\circ} \mathrm{C}$ under agitation at $120 \mathrm{rpm}$. After $24 \mathrm{~h}, 500 \mu \mathrm{L}$ of media in each well was removed and an equal volume of fresh media was added according to each condition (RPMI at pH 5.0 supplemented with $0.5 \%(\mathrm{v} / \mathrm{v})$ acetic acid; RPMI at $\mathrm{pH} 5.0$ supplemented with $0.5 \%(\mathrm{v} / \mathrm{v})$ acetic acid and $1250 \mu \mathrm{g} / \mathrm{mL}$ fluconazole; RPMI at pH 7.0; and RPMI at pH 7.0 supplemented with $1250 \mu \mathrm{g} / \mathrm{mL}$ fluconazole). The concentration of fluconazole was selected according to previous studies with C. glabrata biofilms. ${ }^{16}$ Plates were then incubated for additional $24 \mathrm{~h}$ at $37^{\circ} \mathrm{C}$ under agitation at $120 \mathrm{rpm}$.

\section{RNA isolation}

Following Candida biofilm development, each well was gently washed with $1 \mathrm{~mL}$ of PBS to remove non-adherent cells. Then biofilms were scraped from the plates with $500 \mu \mathrm{L}$ of PBS per well, sonicated for $30 \mathrm{~s}$ at $30 \%$ amplitude (Ultrasonic Processor, Cole-Parmer, IL, USA) in order to separate cells from matrix ${ }^{74}$ and then harvested by centrifugation at $8000 \times g$ for $10 \mathrm{~min}$ at $4^{\circ} \mathrm{C}$. Cells drops were flash frozen in liquid nitrogen and total RNA was isolated from frozen cell pellets using the RiboPure Yeast kit (Ambion, Life Technologies, USA), according to manufacturer's instructions. All samples were treated with Turbo DNAse (Ambion) to remove residual DNA, according to manufacturer's instructions. Total RNA yield was quantified using the Nanodrop 1000 (Thermo Scientific) and RNA quality (RNA Integrity Number (RIN) values $\geq 7.0$ ) assessed on a Bioanalyzer 2100 (Agilent Technologies).

\section{RNA sequencing}

Library preparation and RNA sequencing were performed at Edinburgh Genomics (Scotland, UK). All samples were prepared in biological triplicates and subject to removal of ribosomal RNA before complementary DNA (CDNA) library generation. From these libraries, 50 base-paired-end sequence reads were produced with Illumina Hiseq 2000. The raw sequence data in fastq format as well as the processed data have been deposited in NCBI's Gene Expression Omnibus ${ }^{75}$ and are accessible through GEO Series accession number GSE121074.

\section{Data analysis}

Raw fastq files were successively processed in the following order through Fastqc (v. 10.1), Trimgalore (v. 3.1), Samtools (v. 1.19), Bowtie2 (v. 2.1), and Htseq (v. 5.4). Genome alignment was conducted against the C glabrata CBS138 version s02-m04-r05 chromosomes.fasta file provided by Candida Genome Database. ${ }^{76}$ Aligned data were quality-controlled via the Partek Genomics Suite software (v. 6.6), according to the manufacturer's instructions. Gene expression analysis was performed using Partek Genomics Suite software using a $\log _{2}$ data transformation as the Partek recommended default. Gene ontology (GO) term analysis was performed in parallel through the Candida Genome Database GO Term Finder and the Cytoscape (v. 3) Clue GO plugin. ${ }^{77}$ Network construction was performed with Cytoscape V.3 freeware, ${ }^{78}$ venn diagrams through Venny online freeware (v. 2.0.2) and heatmaps with TM4 MultiExperiment Viewer (MeV, v. 4.9). A statistical comparison among GO term enrichment percentages was performed with GraphPad Prism (v. 6) using the Student's $t$-test for two-tailed data. Data represent three independent biological replicates for each condition.

\section{Quantitative real-time PCR (qPCR)}

Real-Time quantitative PCR of CDNA samples was carried out in a CF X96 Real-Time PCR System from Bio-Rad Laboratories using Power SYBR ${ }^{\circledast}$ Green PCR Master Mix (Applied Biosystems, CA, USA), according to manufacturer's instructions. The High-Capacity cDNA Reverse Transcription Kit
(Invitrogen, CA, USA) was used to synthesize the CDNA in a $20 \mu \mathrm{L}$ reaction containing $1 \mu \mathrm{g}$ of total RNA, according to the manufacturer's instructions. The primers used to amplify the selected genes and the thermocycling conditions are described in Supplementary Table 1. The reaction mixture was set up in a total volume of $20 \mu \mathrm{L}$ using $10 \mu \mathrm{L}$ of SYBR Green PCR Master Mix, $0.3 \mu \mathrm{M}$ of each primer and $4 \mu \mathrm{L}$ of each synthesized CDNA sample (diluted 1:20) and nuclease-free water. A negative control without template was conducted for each gene in each PCR run, and a control for DNA contamination was implemented by using the purified RNA samples as templates. The housekeeping gene, $P G K 1^{79}$ was used to normalize the gene expression. Experiments were performed in triplicate for three independent biological samples. Statistical analysis were performed using two-way ANOVA with Bonferroni correction.

\section{Sterol measurement}

Sterols were extracted according to the method described in Morio et al. ${ }^{80}$ with a few adaptations. In summary, cells were grown for $24 \mathrm{~h}$ in RPMI medium, with or without $24 \mu \mathrm{g} / \mathrm{mL}$ fluconazole. The cells were collected, resuspended in saponification medium, and subjected to vortex mixing. The samples were incubated for $1 \mathrm{~h}$ at $80^{\circ} \mathrm{C}$, after which $1 \mathrm{~mL}$ of water and $4 \mathrm{~mL}$ of hexane were added. After mixing, the two layers were allowed to separate. For spectrophotometrical analysis, UV-transmittable 96-well microtiter plates (Costar Corning) were used to allow measurement of the $\mathrm{OD}_{281}$ and $\mathrm{OD}_{230}$. A formula from Arthington-Skaggs et al. ${ }^{67}$ was used to measure the percentages of ergosterol (corrected for cellular wet weight and resuspension volume). For GC-MS analysis, the sterols were extracted twice with hexane, which was then evaporated by vacuum centrifugation. The sterols were resuspended in $100 \mu \mathrm{L}$ silylating mixture (Sigma) and incubated at room temperature for $30 \mathrm{~min}$. Finally, a corrected cellular wet weight resuspension volume of hexane was added and the samples were immediately stored at $-20^{\circ} \mathrm{C}$ for later analysis by GC-MS. One microliter of the sample was injected into a gas chromatograph-mass spectrometer (Shimadzu QP2010 Ultra Plus) equipped with an HP-5ms nonpolar column (Agilent) (30 $\mathrm{m}$ in length, $0.25-\mathrm{mm}$ inner diameter [id.]; $0.25-\mu \mathrm{m}$ thin layer). Helium was used as carrier gas with a flow rate of $1.4 \mathrm{~mL} / \mathrm{min}$. Injection was carried out at $250^{\circ} \mathrm{C}$ in split mode after $1 \mathrm{~min}$ and with a ratio of $1: 10$. The temperature was first held at $50^{\circ} \mathrm{C}$ for $1 \mathrm{~min}$ and then allowed to rise to $260^{\circ} \mathrm{C}$ at a rate of $50^{\circ} \mathrm{C} / \mathrm{min}$, followed by a second ramp of $2{ }^{\circ} \mathrm{C} / \mathrm{min}$ until $325^{\circ} \mathrm{C}$ was reached; that temperature was maintained for $3 \mathrm{~min}$. The mass detector was operated in scan mode (50 to 600 atomic mass units [amu]), using electron impact ionization $(70 \mathrm{eV})$. The temperatures of the interface and detector were 290 and $250^{\circ} \mathrm{C}$, respectively. A mix of linear $n$-alkanes (from $C_{8}$ to $C_{40}$ ) was injected to serve as external retention index markers. Sterols were identified by their retention time relative to the internal standard (cholestane) and specific mass spectrometric patterns using AMDIS version 2.71. The deconvoluted spectra were matched to GC-MS libraries described in Müller et al..$^{81}$ and NIST/EPA/NIH version 2011. Analysis was performed by integration over the base ion of each sterol, and abundance was calculated relative to the internal standard, comparing the relative peak areas of the compounds across treatments using two-way ANOVA with Bonferroni correction.

\section{Reporting summary}

Further information on research design is available in the Nature Research Reporting Summary linked to this article.

\section{DATA AVAILABILITY}

The datasets generated in this study are available at public repositories or from the corresponding author upon request. The raw RNA-seq data in fastq format, as well as the processed data have been deposited in NCBI's Gene Expression Omnibus and are accessible through GEO Series accession number GSE121074.

Received: 15 October 2019; Accepted: 20 December 2019; Published online: 23 January 2020

\section{REFERENCES}

1. Jermy, A. Stop neglecting fungi. Nat. Microbiol. 2, 17120 (2017).

2. Rodrigues, C., Rodrigues, M., Silva, S. \& Henriques, M. Candida glabrata biofilms: how far have we come? J. Fungi 3, 11 (2017). 
3. Khatib, R., Johnson, L. B., Fakih, M. G., Riederer, K. \& Briski, L. Current trends in candidemia and species distribution among adults: Candida glabrata surpasses $C$. albicans in diabetic patients and abdominal sources. Mycoses 59, 781-786 (2016).

4. Perlin, D. S., Rautemaa-Richardson, R. \& Alastruey-Izquierdo, A. The global problem of antifungal resistance: prevalence, mechanisms, and management. Lancet Infect. Dis. 17, e383-e392 (2017).

5. Silva, S. et al. Adherence and biofilm formation of non-Candida albicans Candida species. Trends Microbiol. 19, 241-247 (2011).

6. Cuellar-Cruz, M. et al. Candida species: new insights into biofilm formation. [Review]. Future Microbiol. 7, 755-771 (2012).

7. Cavalheiro, M. \& Teixeira, M. C. Candida biofilms: threats, challenges, and promising strategies. Front. Med. 5, 28 (2018).

8. Kucharíková, S. et al. In vivo Candida glabrata biofilm development on foreign bodies in a rat subcutaneous model. J. Antimicrob. Chemother. 70, 846-856 (2015).

9. Rodrigues, C. F., Rodrigues, M. E. \& Henriques, M. Susceptibility of Candida glabrata biofilms to echinocandins: alterations in the matrix composition. Biofouling 34, 1-10 (2018).

10. Barelle, C. J. et al. Niche-specific regulation of central metabolic pathways in a fungal pathogen. Cell. Microbiol. 8, 961-971 (2006).

11. Miramón, P. \& Lorenz, M. C. A feast for Candida: Metabolic plasticity confers an edge for virulence. PLoS Pathogens 13, e1006144 (2017).

12. Lorenz, M. C. \& Fink, G. R. The glyoxylate cycle is required for fungal virulence. Nature 412, 83-86 (2001).

13. Osset, J., García, E., Bartolomé, R. M. \& Andreu, A. [Role of Lactobacillus as protector against vaginal candidiasis]. Med. Clínica 117, 285-8 (2001).

14. Owen, D. H. \& Katz, D. F. A vaginal fluid simulant. Contraception 59, 91-95 (1999).

15. Ng, T. S., Desa, M. N. M., Sandai, D., Chong, P. P. \& Than, L. T. L. Growth, biofilm formation, antifungal susceptibility and oxidative stress resistance of Candida glabrata are affected by different glucose concentrations. Infect. Genet. Evol. 40, 331-338 (2016)

16. Mota, S. et al. Candida glabrata susceptibility to antifungals and phagocytosis is modulated by acetate. Front. Microbiol. 6, 919 (2015).

17. Ma, X., Stead, B. E., Rezvanpour, A. \& Davey, M. J. The effects of oligomerization on Saccharomyces cerevisiae Mcm4/6/7 function. BMC Biochem. 11, 37 (2010).

18. Berkow, E. L. \& Lockhart, S. R. Fluconazole resistance in Candida species: a current perspective. Infect. Drug Resistance 10, 237-245 (2017).

19. Vanden Bossche, H. Biochemical targets for antifungal azole derivatives: hypothesis on the mode of action. Curr. Top. Med. Mycol. 1, 313-351 (1985).

20. Nakayama, H., Izuta, M., Nakayama, N., Arisawa, M. \& Aoki, Y. Depletion of the squalene synthase (ERG9) gene does not impair growth of Candida glabrata in mice. Antimicrob. Agents Chemother. 44, 2411-2418 (2000).

21. Jonassen, T. et al. Yeast clk-1 homologue (Coq7/Cat5) is a mitochondrial protein in coenzyme Q synthesis. J. Biol. Chem. 273, 3351-3357 (1998).

22. Ozeir, M. et al. Coq6 is responsible for the C4-deamination reaction in coenzyme Q biosynthesis in Saccharomyces cerevisiae. J. Biol. Chem. 290, 24140-24151 (2015).

23. Johnson, A. et al. COQ9, a new gene required for the biosynthesis of coenzyme $Q$ in Saccharomyces cerevisiae. J. Biol. Chem. 280, 31397-31404 (2005).

24. Turunen, M., Olsson, J. \& Dallner, G. Metabolism and function of coenzyme Q. Biochim. Biophys. Acta-Biomembr. 1660, 171-199 (2004).

25. Eschrich, D., Kötter, P. \& Entian, K. D. Gluconeogenesis in Candida albicans. FEMS Yeast Res 2, 315-325 (2002)

26. Pfanner, N., Wiedemann, N., Meisinger, C. \& Lithgow, T. Assembling the mitochondrial outer membrane. Nat. Struct. Mol. Biol. 11, 1044-1048 (2004).

27. Paschen, Sa, Neupert, W. \& Rapaport, D. Biogenesis of beta-barrel membrane proteins of mitochondria. Trends Biochem. Sci. 30, 575-582 (2005).

28. Kutik, S. et al. Dissecting membrane insertion of mitochondrial beta-barrel proteins. Cell 132, 1011-1024 (2008)

29. Wiedemann, N. et al. Machinery for protein sorting and assembly in the mitochondrial outer membrane. Nature 424, 565-571 (2003).

30. Kozjak, V. et al. An essential role of Sam50 in the protein sorting and assembly machinery of the mitochondrial outer membrane. J. Biol. Chem. 278, 48520-48523 (2003).

31. Milenkovic, D. et al. Sam 35 of the mitochondrial protein sorting and assembly machinery is a peripheral outer membrane protein essential for cell viability. J. Biol. Chem. 279, 22781-22785 (2004).

32. Ishikawa, D., Yamamoto, H., Tamura, Y., Moritoh, K. \& Endo, T. Two novel proteins in the mitochondrial outer membrane mediate beta-barrel protein assembly. $J$. Cell Biol. 166, 621-627 (2004).

33. Meisinger, $C$. et al. The morphology proteins $M d m 12 / M m m 1$ function in the major $\beta$-barrel assembly pathway of mitochondria. EMBO J. 26, 2229-2239 (2007).

34. Fox, E. P. et al. Anaerobic bacteria grow within Candida albicans biofilms and induce biofilm formation in suspension cultures. Curr. Biol. 24, 2411-2416 (2014).
35. Nobile, C. J. et al. A recently evolved transcriptional network controls biofilm development in Candida albicans. Cell 148, 126-138 (2012).

36. Holland, L. M. et al. Comparative phenotypic analysis of the major fungal pathogens Candida parapsilosis and Candida albicans. PLoS Pathog. 10, e1004365 (2014).

37. Zhu, Z. et al. Time course analysis of Candida albicans metabolites during biofilm development. J. Proteome Res. 12, 2375-2385 (2013).

38. Verma-Gaur, J. et al. Integration of posttranscriptional gene networks into metabolic adaptation and biofilm maturation in Candida albicans. PLoS Genet. 11, e1005590 (2015).

39. Calderone, R., Li, D. \& Traven, A. System-level impact of mitochondria on fungal virulence: to metabolism and beyond. FEMS Yeast Res. 15, fov027 (2015).

40. Mora-Montes, H. M. et al. Protein glycosylation in Candida. Future Microbiol. 4, 1167-1183 (2009)

41. Gentzsch, M. \& Tanner, W. Protein-O-glycosylation in yeast: protein-specific mannosyltransferases. Glycobiology 7, 481-486 (1997).

42. Timpel, C., Zink, S., Strahl-Bolsinger, S., Schröppel, K. \& Ernst, J. Morphogenesis, adhesive properties, and antifungal resistance depend on the Pmt6 protein mannosyltransferase in the fungal pathogen Candida albicans. J. Bacteriol. 182, 3063-3071 (2000).

43. Ernst, J. F. \& Prill, S. K. O-glycosylation. Med. Mycol. 39, 67-74 (2001).

44. Prill, S. K. H. et al. PMT family of Candida albicans: five protein mannosyltransferase isoforms affect growth, morphogenesis and antifungal resistance. Mol. Microbiol. 55, 546-560 (2005).

45. Maeda, Y. et al. PIG-M transfers the first mannose to glycosylphosphatidylinositol on the lumenal side of the ER. EMBO J. 20, 250-261 (2001).

46. Fabre, A. L., Orlean, P. \& Taron, C. H. Saccharomyces cerevisiae Ybr004c and its human homologue are required for addition of the second mannose during glycosylphosphatidylinositol precursor assembly. FEBS J. 272, 1160-1168 (2005).

47. Kang, J. Y. et al. PIG-V involved in transferring the second mannose in glycosylphosphatidylinositol. J. Biol. Chem. 280, 9489-9497 (2005).

48. Fiori, A. et al. The heat-induced molecular disaggregase Hsp104 of Candida albicans plays a role in biofilm formation and pathogenicity in a worm infection model. Eukaryot. Cell 11, 1012-1020 (2012).

49. Gillies, A. T., Taylor, R. \& Gestwicki, J. E. Synthetic lethal interactions in yeast reveal functional roles of J protein co-chaperones. Mol. Biosyst. 8, 2901-2908 (2012).

50. Gong, Y., Li, T., Yu, C. \& Sun, S. Candida albicans heat shock proteins and Hspsassociated signaling pathways as potential antifungal targets. Front. Cell. Infect. Microbiol. 7, 520 (2017).

51. Yoo, J. Il et al. Proteomic analysis of cellular and membrane proteins in fluconazole-resistant candida glabrata. Osong Public Heal. Res. Perspect. 3, 74-78 (2012).

52. Cowen, L. E. \& Lindquist, S. Hsp90 potentiates the rapid evolution of new traits: drug resistance in diverse fungi. Science (80-.) 309, 2185-2189 (2005).

53. Cowen, L. E., Carpenter, A. E., Matangkasombut, O., Fink, G. R. \& Lindquist, S. Genetic architecture of Hsp90-dependent drug resistance. Eukaryot. Cell 5, 2184-2188 (2006).

54. Cormack, B. P., Ghori, N. \& Falkow, S. An adhesin of the yeast pathogen Candida glabrata mediating adherence to human epithelial cells. Science 285, 578-582 (1999).

55. Vale-Silva, L. A. et al. Upregulation of the adhesin gene EPA1 mediated by PDR1 in Candida glabrata leads to enhanced host colonization. mSphere 1, 1-16 (2016).

56. Srivastava, V. K., Suneetha, K. J. \& Kaur, R. A systematic analysis reveals an essential role for high-affinity iron uptake system, haemolysin and CFEM domaincontaining protein in iron homeostasis and virulence in Candida glabrata. Biochem. J. 463, 103-114 (2014).

57. Martínez-Duncker, I., Díaz-Jímenez, D. F. \& Mora-Montes, H. M. Comparative analysis of protein glycosylation pathways in humans and the fungal pathogen candida albicans. Int. J. Microbiol. 2014, 267497 (2014).

58. Lussier, M., Sdicu, A. M., Bussereau, F., Jacquet, M. \& Bussey, H. The Ktr1p, Ktr3p, and Kre2p/Mnt1p mannosyltransferases participate in the elaboration of yeast Oand N-linked carbohydrate chains. J. Biol. Chem. 272, 15527-15531 (1997).

59. Ene, I. V. et al. Host carbon sources modulate cell wall architecture, drug resistance and virulence in a fungal pathogen. Cell. Microbiol. 14, 1319-1335 (2012).

60. Ene, I. V. et al. Carbon source-induced reprogramming of the cell wall proteome and secretome modulates the adherence and drug resistance of the fungal pathogen Candida albicans. Proteomics 12, 3164-3179 (2012).

61. Ballou, E. R. et al. Lactate signalling regulates fungal $\beta$-glucan masking and immune evasion. Nat. Microbiol 2, 16238 (2016).

62. Rodriguez, C. R. et al. Kin28, the TFIIH-associated carboxy-terminal domain kinase, facilitates the recruitment of mRNA processing machinery to RNA polymerase II. Mol. Cell. Biol. 20, 104-112 (2000).

63. Alghamdi, T. A. et al. Vac14 protein multimerization is a prerequisite step for Fab1 protein complex assembly and function. J. Biol. Chem. 288, $9363-9372$ (2013). 
64. Fiori, A. \& Van Dijck, P. Potent synergistic effect of doxycycline with fluconazole against Candida albicans is mediated by interference with iron homeostasis. Antimicrob. Agents Chemother. 56, 3785-3796 (2012).

65. Demuyser, L. et al. Mitochondrial cochaperone Mge1 is involved in regulating susceptibility to fluconazole in Saccharomyces cerevisiae and Candida species. MBio. 8, e00201-e00217 (2017).

66. Prasad, T., Chandra, A., Mukhopadhyay, C. K. \& Prasad, R. Unexpected link between iron and drug resistance of Candida spp.: iron depletion enhances membrane fluidity and drug diffusion, leading to drug-susceptible cells. Antimicrob. Agents Chemother. 50, 3597-3606 (2006).

67. Arthington-Skaggs, B. A., Jradi, H., Desai, T. \& Morrison, C. J. Quantitation of ergosterol content: Novel method for determination of fluconazole susceptibility of Candida albicans. J. Clin. Microbiol. 37, 3332-3337 (1999).

68. Rodrigues, C. F., Silva, S. \& Henriques, M. Candida glabrata: a review of its features and resistance. Eur. J. Clin. Microbiol. Infect. Dis. 33, 673-88 (2014).

69. Brown, A. J. P., Brown, G. D., Netea, M. G. \& Gow, N. A. R. Metabolism impacts upon Candida immunogenicity and pathogenicity at multiple levels. Trends Microbiol. 22, 614-622 (2014).

70. Mortensen, P. B. \& Clausen, M. R. Short-chain fatty acids in the human colon: relation to gastrointestinal health and disease. Scand. J. Gastroenterol. Suppl. 216, 132-148 (1996).

71. Hameed, S. et al. Iron deprivation induces EFG1-mediated hyphal development in Candida albicans without affecting biofilm formation. FEMS Yeast Res. 8, 744-755 (2008)

72. Ramage, G. Investigation of multidrug efflux pumps in relation to fluconazole resistance in Candida albicans biofilms. J. Antimicrob. Chemother. 49, 973-980 (2002).

73. Mathé, L. \& Van Dijck, P. Recent insights into Candida albicans biofilm resistance mechanisms. Curr. Genet. 59, 251-264 (2013).

74. Fonseca, E. et al. Effects of fluconazole on Candida glabrata biofilms and its relationship with $A B C$ transporter gene expression. Biofouling 30, 447-57 (2014).

75. Edgar, R., Domrachev, M. \& Lash, A. Gene Expression Omnibus: NCBI gene expression and hybridization array data repository. Nucl. Acids Res. 30, 207-210 (2002).

76. Inglis, D. O. et al. The Candida genome database incorporates multiple Candida species: Multispecies search and analysis tools with curated gene and protein information for Candida albicans and Candida glabrata. Nucl. Acids Res. 40, 667-674 (2012).

77. Bindea, G. et al. ClueGO: a Cytoscape plug-in to decipher functionally grouped gene ontology and pathway annotation networks. Bioinformatics 25, 1091-1093 (2009).

78. Shannon, P. et al. Cytoscape: a Software Environment for Integrated Models of Biomolecular Interaction Networks. Genome Res. 13, 2498-2504 (2003).

79. Li, Q. Q., Skinner, J. \& Bennett, J. E. Evaluation of reference genes for real-time quantitative PCR studies in Candida glabrata following azole treatment. BMC Mol. Biol. 13, 22 (2012).

80. Morio, F., Pagniez, F., Lacroix, C., Miegeville, M. \& Le pape, P. Amino acid substitutions in the Candida albicans sterol 85,6 -desaturase (Erg3p) confer azole resistance: characterization of two novel mutants with impaired virulence. $J$. Antimicrob. Chemother. 67, 2131-2138 (2012).

81. Müller, C., Binder, U., Bracher, F. \& Giera, M. Antifungal drug testing by combining minimal inhibitory concentration testing with target identification by gas chromatography-mass spectrometry. Nat. Protoc. 12, 947-963 (2017).

\section{ACKNOWLEDGEMENTS}

This study was supported by the Portuguese National Funding Agency for Science, Research, and Technology FCT (grant PTDC/BIAMIC/5184/2014). R.A. received FCT Ph. D fellowship (PD/BD/113813/2015). We gratefully acknowledge Edinburgh Genomics for RNA-Seq library preparation and sequencing. The work on CBMA was supported by the strategic programs UID/BIA/04050/2013 (POCI-01-0145-FEDER-007569) and UID/BIA/04050/2019. The work on CEB was supported by PEst-OE/EQB/LA0023/2013, from FCT, "BioHealth-Biotechnology and Bioengineering approaches to improve health quality", Ref. NORTE-07-0124-FEDER-000027, co-funded by the Programa Operacional Regional do Norte (ON.2-O Novo Norte), QREN, FEDER and the project "Consolidating Research Expertize and Resources on Cellular and Molecular Biotechnology at CEB/IBB", Ref. FCOMP-01-0124-FEDER-027462. The work in Aberdeen was also supported by the European Research Council through the advanced grant "STRIFE" (C-2009-AdG-249793), by the UK Medical Research Council (MR/M026663/1) and by the Medical Research Council Center for Medical Mycology and the University of Aberdeen (MR/N006364/1). The work at KU Leuven was supported by the Federation of European Biochemical Societies (FEBS) through a short-term fellowship awarded to R.A. and by the Fund for Scientific Research Flanders (FWO; WO.009.16N). We thank Beatriz Herrera-Malaver for technical assistance with the GC-MS analysis.

\section{AUTHOR CONTRIBUTIONS}

S.P., R.A., M.C., M.H., S.S., P.V.D. and A.J.P.B. conceived and designed the experimental studies. R.A., S.L.K., A.G., N.A., C.F.R. and L.D. performed the experiments. R.A. and S.P. wrote the manuscript with additional input from M.H., S.S., M.C., L.D., P.V.D. and A.J.P.B. All authors reviewed and approved the manuscript.

\section{COMPETING INTERESTS}

The authors declare no competing interests.

\section{ADDITIONAL INFORMATION}

Supplementary information is available for this paper at https://doi.org/10.1038/ s41522-020-0114-5.

Correspondence and requests for materials should be addressed to S.P.

Reprints and permission information is available at http://www.nature.com/ reprints

Publisher's note Springer Nature remains neutral with regard to jurisdictional claims in published maps and institutional affiliations.

Open Access This article is licensed under a Creative Commons Attribution 4.0 International License, which permits use, sharing, adaptation, distribution and reproduction in any medium or format, as long as you give appropriate credit to the original author(s) and the source, provide a link to the Creative Commons license, and indicate if changes were made. The images or other third party material in this article are included in the article's Creative Commons license, unless indicated otherwise in a credit line to the material. If material is not included in the article's Creative Commons license and your intended use is not permitted by statutory regulation or exceeds the permitted use, you will need to obtain permission directly from the copyright holder. To view a copy of this license, visit http://creativecommons. org/licenses/by/4.0/.

(c) The Author(s) 2020 